\title{
Las ferias libres y el problema de las subsistencias: Santiago de Chile, 1939-1943
}

\section{The Farmer's Market and the Subsistence Problem: Santiago of Chile, 1939-1943}

\author{
Juan Carlos Yáñez Andrade \\ Universidad de Valparaíso \\ juancarlos.yanez@uv.cl \\ DOI: I0.2490I/rehs.v40iI57.309
}

\section{(c) (i) (8)}

Las ferias libres y el problema de las subsistencias: Santiago de Chile, I939-1943 por Juan Carlos Yañez Andrade se distribuye bajo una Licencia Creative Commons Atribución-NoComercial 4.0 Internacional.

Fecha de recepción: 27 de mayo de 2017

Fecha de aprobación: 29 de noviembre de 2017

\section{RESUMEN:}

Este artículo analiza la crisis del sistema de abastos de la ciudad de Santiago de Chile en el contexto de la Gran Depresión. Se estudian los debates generados en torno al alza en el costo de la vida y las propuestas de creación de las ferias libres por parte de la administración de la alcaldesa Graciela Contreras en 1939. Además, se presentan las distintas luchas y modalidades de organización que se dieron durante el periodo.

Palabras clave:

Ferias libres, alimentación, políticas alimentarias, subsistencias, Chile.

\section{ABSTRACT:}

This article analyzes the crisis of the supply system in the city of Santiago de Chile in the context of the Great Depression, by examining the debates generated by the rising cost of living and proposals to create a series of farmer's markets that emerged in 1939 during the administration of Mayor Graciela Contreras. The study includes a discussion of the different struggles and organizational modalities that characterized this period of the city's history. 
Keywords:

Farmer's markets, alimentation, food policies, subsistence, Chile.

Los avances de las últimas décadas en la historia de la salud y de la ciencia han abierto opciones interesantes para continuar conociendo sobre las políticas sanitarias de América Latina. Provechosas han sido las investigaciones que han vinculado la formulación de un conjunto de políticas públicas con la eclosión de la llamada "cuestión social" y el mejoramiento nutricional de los sectores populares. ${ }^{2}$ En especial, los estudios sobre alimentación popular se han centrado en las modalidades del acceso de los alimentos en grupos sociales específicos, como forma de mejorar los rendimientos productivos de la nación o promover un ideal de trabajador. ${ }^{3}$ Las perspectivas transnacionales han puesto hincapié en la necesidad de pensar la alimentación como un problema social y objeto de políticas públicas durante el periodo de entre guerra, en especial a partir del desarrollo de instancias internacionales y panamericanas de circulación de ideas. ${ }^{4}$

En el caso de Chile, los diagnósticos sobre los problemas de alimentación que sufría la población, en particular la de escasos recursos, databan de fines del siglo XIX, aunque sólo a comienzos de la década de 1930 se avanzó en la implementación de una política alimentaria. En i93i se llevó a cabo el Primer Congreso de Alimentación Popular y en 1937 fue creado el Consejo Nacional de Alimentación, encargado de asesorar al gobierno y proponer medidas legislativas que mejoraran la condición nutricional de la población. Muchos sectores responsabilizaron al alza en el costo de la vida como la causa principal de las dificultades para acceder a los artículos de primera necesidad, sumada a la crisis de abastecimiento de la ciudad de Santiago, el cual se llevaba a cabo en el Mercado Central, la Vega Municipal y el Matadero. Para las autoridades se hacía imperativo implementar una serie de medidas que acercaran los alimentos a la población, una de las cuales era la creación de las ferias libres, rompiendo de esta forma con el monopolio que tenía el sistema tradicional de abastos.

Así como las políticas de alimentación han recibido muy poco interés por parte de la historiografía chilena, las modalidades de distribución de alimentos también son poco conocidas, por lo que existen escasas investigaciones que hayan estudiado específicamente las ferias libres. En I955 una memoria de prueba abordó su creación en el marco de la llegada al poder del Frente Popular en 1938 y la implementación de una política de disminución del costo de la vida. ${ }^{6}$ El año 2003, el historiador Gabriel Salazar publicó un estudio sobre las ferias libres que puede ser considerado uno de los más completos y que a borda su desarrollo desde la época colonial, reivindicando estos espacios como instancias de soberanía popular. ${ }^{7}$ Para Salazar, el comercio popular o "regatón" habría sido el espacio que tuvieron las masas empobrecidas durante el siglo XIX para desarrollar su inclinación empresarial, una vez cortados los circuitos del emprendimiento minero, agrícola y artesanal. Si bien Salazar aborda la crisis del sistema de abastos de la ciudad de Santiago, la que se hizo manifiesta con la Gran Depresión, y el rol que comienzan a jugar las ferias en la oferta de alimentos, no profundiza en los debates sobre su instalación, ni en la evaluación que se hizo de ellas durante el momento clave de su institucionalización bajo la gestión de la alcaldesa Graciela Contreras de Schnake (1939-1940). 
Es en torno al debate sobre las ferias libres y la crisis de abastecimiento, que sufre la capital durante la década de 1930, que se inserta el presente artículo. Aunque las propuestas de creación pueden rastrearse hasta comienzos del siglo XX, fue en el año I939 que se aprobó de manera formal el establecimiento de las primeras ferias libres. Formulamos como hipótesis central que esta medida se vio reforzada, primero, por los diagnósticos críticos que se hicieron del antiguo sistema de abastos y, segundo, por el nombramiento de Graciela Contreras en la alcaldía de Santiago, quien levantó un programa progresista que tuvo en la alimentación uno de sus pilares fundamentales.

El artículo se estructura en tres secciones. La primera busca explicar las modalidades de funcionamiento del sistema de abastos de la ciudad de Santiago, y las crecientes críticas que comenzó a recibir a partir de comienzos de la década de i930. Posteriormente se analiza el funcionamiento de las primeras ferias libres y los debates en torno a su utilidad como solución a los problemas de abastecimiento. Por último, describimos las modalidades de organización y de lucha que se dieron en torno a la creación de las ferias libres, poniendo especial atención en la defensa que de ellas hicieron los sectores populares.

Las fuentes utilizadas son variadas, partiendo por la prensa nacional y municipal, las que permiten rastrear el debate en torno a la creación de las ferias libres, junto con las sesiones de la Municipalidad de Santiago donde se formularon las respectivas reglamentaciones, finalizando con el movimiento político y gremial que llevaron a cabo los feriantes a partir de i939 con el objetivo de que se reconociera su actividad.

\section{El sistema de abastos del Santiago tradicional}

A comienzos de la década de i930, Chile sufrió las consecuencias de la Gran Depresión, en el contexto de la restricción del crédito externo y la paralización de las faenas productivas, presentando hacia I932 un elevado desempleo, que según algunos cálculos alcanzó el I5\% de la población activa, es decir, I90,ooo cesantes..$^{8}$ La ciudad de Santiago fue la más afectada por la crisis, abriendo albergues y ollas de pobres para atender a la población proveniente de las oficinas salitreras del norte del país, a lo que hay que agregar la incapacidad de las autoridades en resolver los problemas de alimentación y de vivienda provocados por el crecimiento poblacional. Entre los censos de I930 y I940, la capital pasó de una población de 836,928 habitantes a una de I,IOo,725, siendo la comuna de Santiago, sector donde se localizaban los principales núcleos administrativos del Estado, la que concentró la mayor tasa de crecimiento, con cerca de roo mil personas. ${ }^{2}$ El comercio, en particular el informal, fue uno de los pocos sectores dinamizadores de la economía y ámbito donde muchos desempleados buscaron la manera de ganarse la vida, pasando de ı09,000 a I62,000 trabajadores, lo que correspondía a un $9.2 \%$ de la población activa. $\stackrel{\text { 우 }}{ }$

De esta forma, el crecimiento y concentración de la población en Santiago, sumado a los efectos de la crisis económica, configuró un cuadro de crisis social y política que las autoridades del gobierno de Arturo Alessandri (1932-1938) buscaron enfrentar a través de un plan heterodoxo 
de control de precios, fomento de obras públicas, regulación de la sindicalización, inauguración de albergues y promoción de actividades recreativas para los sectores populares.

El aspecto más palpable de la crisis fue el alza sostenida en el costo de la vida, especialmente en el ítem de alimentos. Diversas encuestas de nutrición habían determinado la importancia que tenía la alimentación en la estructura de gastos de las familias más modestas, estableciendo que cerca de un $70 \%$ de sus ingresos se destinaban a este rubro. ${ }^{\text {II }}$ Dentro de ellos el consumo de trigo, especialmente en la forma de pan, representaba casi el $50 \%$ de la ingesta calórica diaria, a lo que había que agregar las papas, legumbres y hortalizas como base de la comida popular. En cuanto a la carne, su consumo se restringía a algunos gramos diarios (entre 50 y roo gramos), representando sólo el io \% del aporte calórico total. $\underline{\text { I2 }}$

Como se muestra en la gráfica I, la inflación tuvo un incremento constante durante la década de 1930 , pasando de un índice de Ioo puntos en marzo de 1928 a I84 en diciembre de I938, correspondiendo la mayor alza a los alimentos, aunque las cifras pueden ser mayores si consideramos que la Dirección General de Estadística le asignó a este rubro solo un $45 \%$ del total de la canasta.

Gráfica I. Índice del costo de la vida

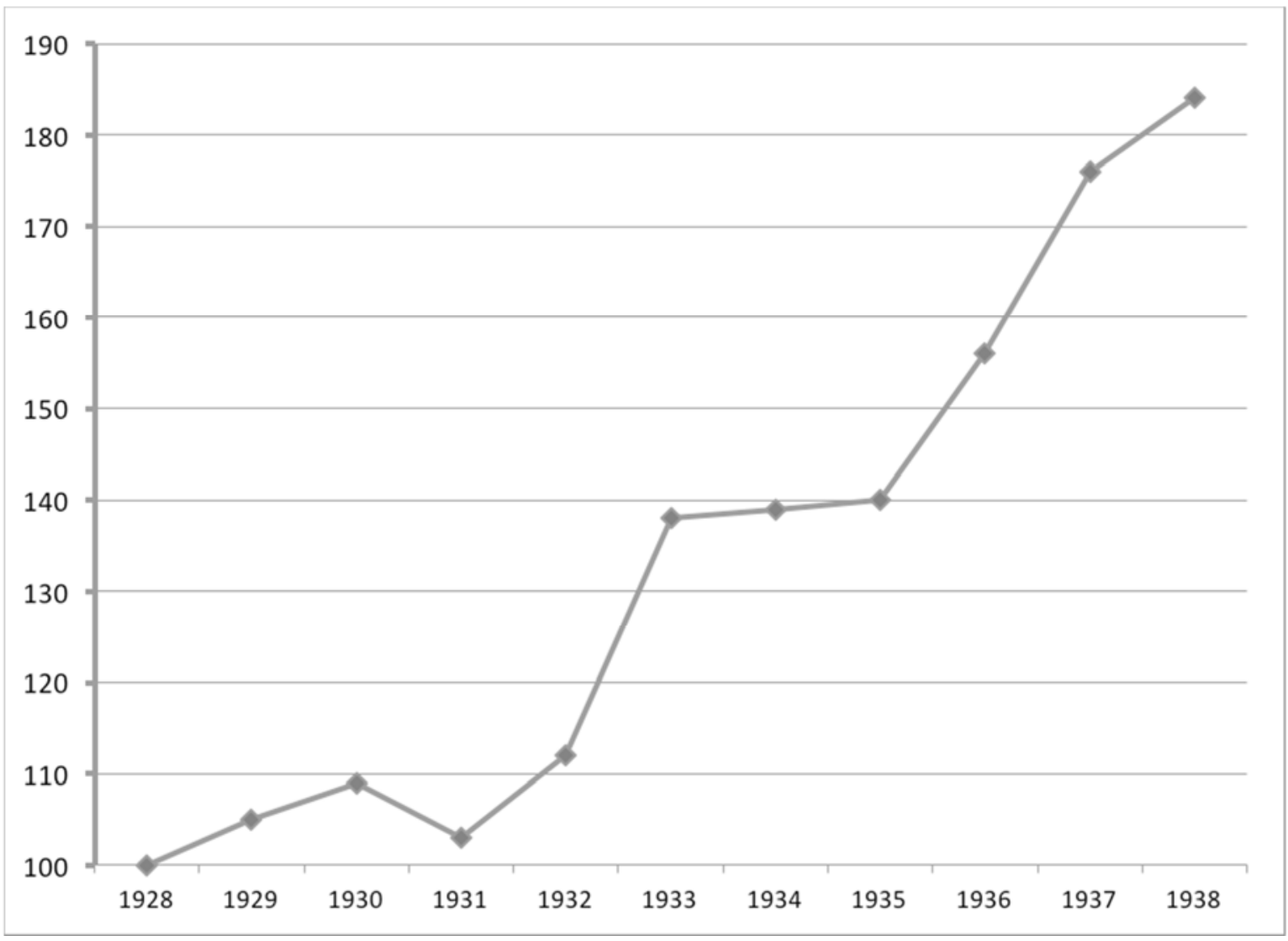


Fuente: Elaboración propia a partir de Estadística Chilena (I2) (diciembre 1938). *marzo I $928=\mathrm{IOO}$.

Muchos responsabilizaron al sistema de abastos por el alza en el costo de los alimentos, centrándose las críticas especialmente en la Feria o Vega Municipal (también llamada Central), ubicada en la ladera norte del río Mapocho, y que actuaba como el principal punto de abastecimiento de frutas y verduras de la ciudad. Los cuestionamientos se dirigían, más específicamente, a la presencia de los consignatarios, encargados de la venta al por mayor y quienes -según denunciaba la prensa- subían artificialmente los precios a través del control de la venta en privado, disminuyendo con ello los remates de productos en oferta pública, tal como la ley lo establecía. ${ }^{13}$ La situación se agravó a tal punto que en junio de i94I el Comisariato de Subsistencia y Precios, organismo público regular en materia económica, ${ }^{14}$ aprobó una norma que obligaba a que todas las verduras y hortalizas de la Vega se vendieran en remate, autorizando al vendedor mayorista a tener una utilidad máxima de un $5 \%$ en relación con el costo bruto y al detallista de un $20 \%$, valores que incluían las comisiones de martilleros, los créditos y derechos municipales. 5

La prensa de distinto signo ideológico se hizo eco de los problemas de desabastecimiento y alza de los precios durante los treinta, mostrándose especialmente crítica por la especulación que se daba en la venta de las papas, producto eminentemente popular y base de la comida de los trabajadores. ${ }^{16}$ Si bien desde 1932 existía el Comisariato de Subsistencia y Precios, el cual llevaba a cabo una labor permanente de fiscalización y de requisamiento de productos de primera necesidad, muchos sectores criticaron que el gobierno no tuviera una política más integral para enfrentar el desabastecimiento. ${ }^{17}$ En particular se cuestionó la ausencia de medidas que incentivaran la producción agrícola y mejoraran las condiciones de almacenamiento y distribución de los productos.

En cuanto a las papas, si bien en algunos años se observa un crecimiento de su producción, el alza en los precios internacionales de los productos agrícolas y la política monetaria del ministro de Hacienda Gustavo Ross, estimularon su exportación, agravando aún más las condiciones desfavorables del mercado interno, tal como ocurrió en 1936 cuando se exportaron i2,00o,ooo de kilos durante el primer semestre, cifra extraordinaria en comparación con los 500,ooo kilos del mismo periodo del año anterior. $\frac{18}{}$

La exportación de papas tuvo efectos perjudiciales para el stock disponible en la Vega Municipal, disminuyendo progresivamente sus entradas de 354,000 sacos de 8 o kilos en i931 a 26r,ooo en I935. ${ }^{19}$ De esta forma, y pese a eventuales aumentos de producción, el precio de las papas se mantuvo al alza durante toda la década de i930, lo que obligó a la Municipalidad de Santiago a denunciar en el Comisariato de Subsistencia y Precios a los especuladores, dentro de los cuales se encontraban los consignatarios que reducían, como se dijo anteriormente, su venta en remate público.e. Como lo muestra la gráfica 2, su precio pasó de \$0.2I en I93I a \$o.69 en I940, con un aumento cercano al $300 \%$ en diez años. 
Gráfica 2. Evolución precio de las papas en la Vega Municipal

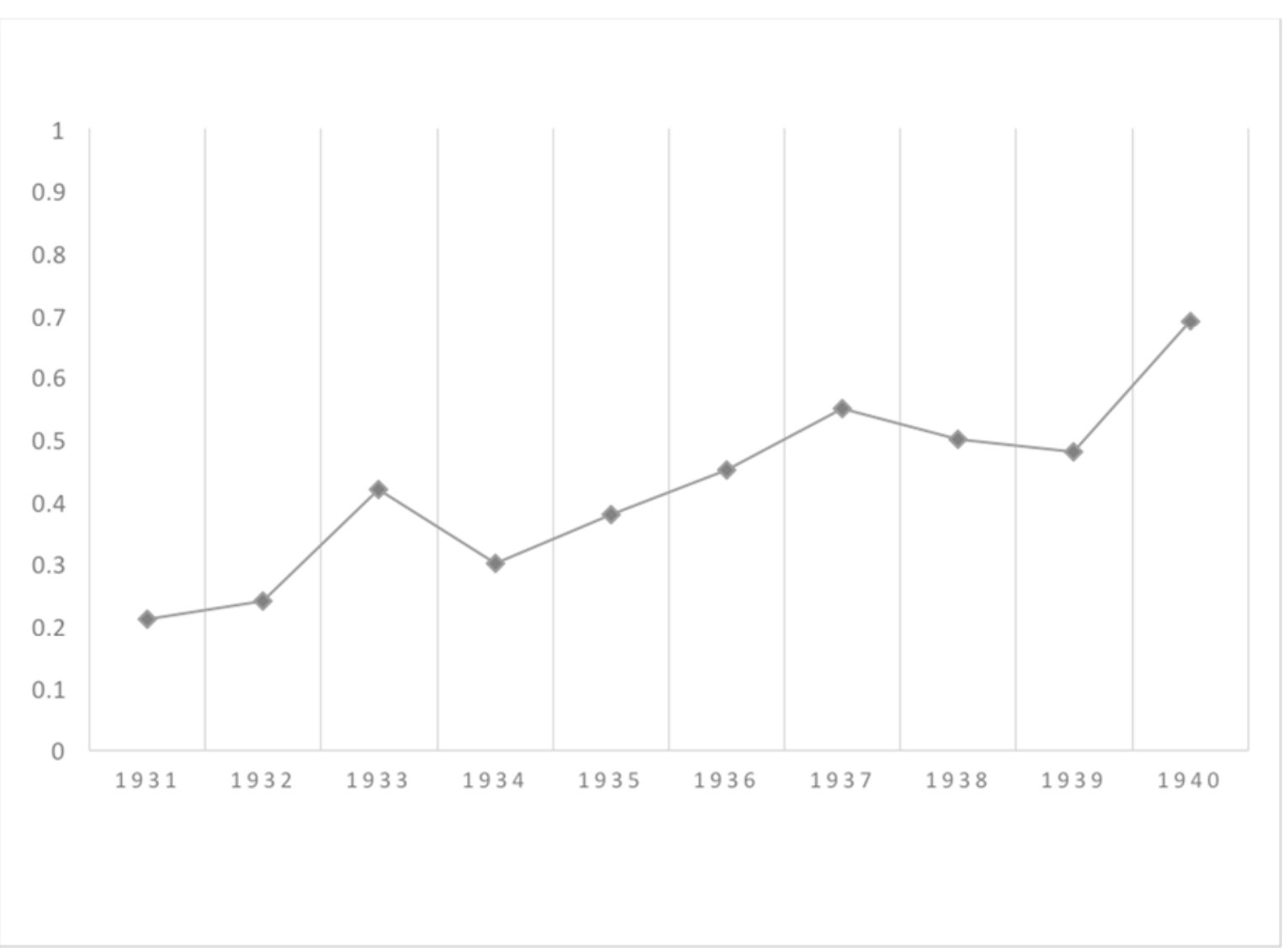

Fuente: elaboración propia a partir de Boletín Municipal, núm. 3,158 y núm. 4,808; La Opinión, 7 de abril de 1937. Se ha tomado como referencia el precio de las papas de primera categoría.

Frente a esta alza sostenida en el costo de los alimentos y la serie de reclamos que se sucedieron desde 1935 sobre el funcionamiento del sistema de abastos, la Municipalidad de Santiago abordó en el mes de marzo de 1936 la modificación del Reglamento de Mercados y Ferias Municipales. ${ }^{21}$ El debate se polarizó entre aquellos sectores que apuntaban al rol que podía desarrollar el municipio en favorecer la venta de productos agrícolas, bajando con ello los precios, y los que señalaban que el aumento en el costo de la vida se debía más a razones estructurales difíciles de abordar a través de simples normativas municipales. En este último aspecto se hacía referencia al problema de tenencia de la tierra y el precario sistema de transporte y distribución existente..$^{22}$

En julio de 1936, el concejo municipal discutió un informe de su Departamento de Control y Estadística, el cual criticaba el débil poder regulador que tenía el municipio en la distribución y venta de alimentos, así como las inversiones realizadas en el mejoramiento de los mercados de la comuna sin tener en cuenta una política integral sobre el problema de las subsistencias. ${ }^{23}$ Los mercados -señalaba el informe- debían reorganizarse para que los productos de primera 
necesidad fueran ofrecidos en subasta pública y proveídos por la respectiva circunscripción municipal. Además, debían especializarse en minoristas y mayoristas, distribuidos en la ciudad de acuerdo a consideraciones sanitarias y densidad de población.

El concejo municipal, en sesión del 3 de septiembre de 1936, resolvió modificar el Reglamento de Mercados y Ferias Municipales que databa de 1933, dando cuenta del nivel de preocupación que tenían las autoridades por el funcionamiento del sistema de abastos. Se aprobó la supresión de las ventas en privado de frutas y verduras, así como la eliminación de los consignatarios y la futura reducción de un $50 \%$ de los locatarios de las ferias y mercados. ${ }^{24}$ Con estas medidas se buscaba tener un mayor control en la fluctuación de los precios, ordenando, además, las modalidades de venta, con lo cual se reconocía que el aumento de los permisos no había significado una disminución en el valor de los productos. Sin embargo, los comerciantes se opusieron, argumentando que ninguna de estas acciones resolvía los problemas de fondo en el funcionamiento del sistema de abastos de la capital. Por ejemplo, el Sindicato de Comerciantes en Subsistencias de la Vega Matadero llamó a crear un "consejo técnico y gremial de subsistencia" que formulara un plan general al problema del costo de la vida, ${ }^{25}$ en tanto que la Asociación de Productores de Chacarería, Frutas y Hortalizas de Santiago criticó estos acuerdos por ser contraproducentes al no ir acompañados de una reorganización profunda en el funcionamiento de ferias y mercados, planteando la necesidad de dejar de ver a la Vega Central -principal centro de distribución de frutas y verduras- como un lugar de explotación para transformarlo "en un medio público de facilitar las relaciones entre el productor y el consumidor". ${ }^{26}$ Demandaba, concretamente, la disminución de impuestos, amenazando que de no ser aceptada esta solicitud prescindiría de los servicios municipales, vendiendo directamente sus productos a los consumidores. El alcalde de Quilicura, comuna rural del norte de la capital, se manifestó en el mismo sentido, anunciando la posible creación, junto a los alcaldes de Colina, Conchalí, Barrancas y Maipú, de una Vega fuera de la circunscripción de Santiago, "donde el productor verá resguardados sus derechos y donde el consumidor pueda adquirir a mejor precio y calidad". ${ }^{27}$ Si bien estas resoluciones del concejo municipal no se llevaron a cabo al ser paralizadas por el Comisariato de Subsistencias y Precios, los debates generados en torno a un nuevo reglamento de ferias y mercados mostraban la complejidad de los intereses en juego.

Un aspecto no menor, y que explica las dificultades de las autoridades para ejercer un efectivo control en el funcionamiento de mercados y ferias, es la importancia económica que tenía este comercio en el presupuesto municipal, por lo que cualquier limitación de sus actividades o, directamente, disminución en el número de locatarios, reduciría las entradas del municipio.

Con el fin de poner en perspectiva la relevancia de este tipo de comercio, podemos señalar que para la segunda mitad de la década de 1930 los ingresos municipales por concepto de explotación -lo que incluía el arriendo de espacios en ferias y mercados-alcanzaron en promedio la suma de 590,000 pesos mensuales, algo superior a los 500,000 pesos que se obtenían por los impuestos directos (de alcoholes, vehículos y territoriales, entre otros), siendo la Feria Municipal la que obtenía, por sí sola, una cifra cercana a 270,00o mensuales (46\% del total aproximadamente), tal como se observa en la tabla . 
Tabla I. Ingresos mensuales Municipalidad de Santiago (1938)

\begin{tabular}{|c|c|c|c|}
\hline Explotaciones & Ingresos & Impuestos & Ingresos \\
\hline Arriendo Feria Municipal & $270,139.65$ & Impuesto territorial & $315,560.54$ \\
\hline Arriendo Mercado Central & $5^{1}, 846.70$ & Impuesto de Matadero & $77,484.60$ \\
\hline Arriendo Mercado Matadero & $6 \mathrm{I}, 65 \mathrm{I} .8 \mathrm{o}$ & Patentes de perro & $55^{\circ}$ \\
\hline Arriendo Mercado Poniente & $9,003.16$ & Patentes de vehículo & I,9I5.83 \\
\hline Derechos remate de llaves & I,797 & Morosos patente & $80,876.95$ \\
\hline Entradas de Matadero & $22,622.90$ & Patente de alcoholes & 30,275 \\
\hline Arriendo Cancha Matanza & $\mathrm{I}, 4 \mathrm{IO}$ & & \\
\hline Recaudación de aseo & $\mathrm{I} 62,654.80$ & & \\
\hline Boleterías Cerro Santa Lucía & 6,47I.6o & & \\
\hline Boleterías Parque Cousiño & $2,847.80$ & & \\
\hline Total & $590,445 \cdot 4 \mathrm{I}$ & & $506,662.9^{2}$ \\
\hline
\end{tabular}

Fuente: Elaboración propia a partir de Boletín Municipal, núm. 3,979. Se ha tomado como mes de referencia a noviembre de 1938

De esta forma, las disputas que se dieron a partir de 1939 en torno a la creación de las ferias libres fueron expresión de los intereses que cruzaban a los diversos grupos vinculados al comercio, la ciudadanía y al mismo municipio.

Ferias libres: ¿una solución definitiva?

Las ferias libres no surgieron en la capital en 1939 con el Frente Popular y la administración de la alcaldesa Graciela Contreras. Desde comienzos del siglo XX, los municipios habían autorizado, a modo de ensayo, su instalación con el fin de ofrecer nuevos lugares de expendio de alimentos y detener en parte el alza en el costo de la vida, aunque no pasaron de ser experiencias acotadas, faltas de todo reglamento y que recibieron diversas críticas. Por ejemplo, con ocasión del Primer Congreso de Alimentación Popular, desarrollado en Valparaíso en I93i, el médico 
Edwyn Reed criticó las ferias francas del puerto por los graves problemas sanitarios que provocaban, señalando que tanto los productos como los vendedores no eran sometidos a ningún tipo de inspección. $\underline{28}$

Con la administración de Oscar Ruiz Tagle en la alcaldía de Valparaíso (1935-1938) hubo intenciones claras de suprimir las ferias libres y el comercio ambulante, aduciendo razones de orden público y de ataque a la especulación. ${ }^{29}$ Los feriantes y vendedores ambulantes recibieron el apoyo del diputado socialista Amaro Castro $^{30} y$ de la Unión de Feriantes de Valparaíso, ${ }^{\text {,I }}$ quienes denunciaron a los grandes comerciantes por su supuesto interés en eliminarlas. En este sentido, la Unión de Feriantes de Valparaíso señaló lo siguiente:

Que los intereses creados que nos combaten reconocen públicamente en sus campañas que el mantenimiento de las ferias libres les ocasiona una competencia desleal, causa por supuesto que beneficia enormemente a los consumidores que en número de 30,000 que a ella concurren a aprovecharse y proveerse de los artículos que allí se expenden. ${ }^{32}$

Además, demandó la aprobación de un reglamento para las ferias libres, que contemplara, por una parte, fuertes castigos a quienes infringieran sus disposiciones y, por otra, que reconociera la colaboración de sus inspectores ad honorem, los cuales velaban "por los intereses de los consumidores y la debida cultura que es el baluarte de la organización". 33 Esta declaración es interesante porque muestra cómo los feriantes buscaron el reconocimiento por parte de las autoridades y la formalización de sus actividades, dándose para estos fines una organización gremial que tuvo vinculaciones con congresistas y federaciones sindicales.

En Santiago, los vendedores ambulantes y feriantes informales tuvieron menos posibilidades de encontrar espacios para instalar sus puestos de venta por la existencia de un mayor control policial y porque los intereses de los grandes comerciantes y el municipio eran más fuertes que en Valparaíso. Luego del funcionamiento provisorio de una feria libre en I9I5, el Departamento de Subsistencia de la Municipalidad de Santiago organizó una en i935 en la calle Manuel Montt, sector de Exposición cercana a la Estación Central de Ferrocarriles, a la cual concurrirían a vender sus productos los chacareros de los alrededores de Santiago. ${ }^{34}$ Además, la prensa informó sobre la instalación de una feria libre en el sector del Barrio Matadero, sur de la capital, y otra dependiente de la Municipalidad de Nuñoa, en la zona oriente, contigua al Mercado Municipal (calle Irarrázaval).

Sin embargo, es con la designación de Graciela Contreras como alcaldesa de Santiago que se intentó abordar de manera más integral el problema del abastecimiento de la capital. ${ }^{35}$ Una vez nombrada por el recién electo presidente de la República, Pedro Aguirre Cerda (I938-I94I), buscó implementar una agenda de política social que tuvo en la búsqueda de bienestar del pueblo su preocupación central. En una de sus primeras entrevistas resumió su programa de gobierno en la necesidad de educar al pueblo, entregando principios de moralidad, para lo cual era fundamental erradicar los conventillos, construir plazas de juego para los niños, fomentar el 
tiempo libre de los trabajadores y sus familias, y asegurar su alimentación con un restaurante y hospedería municipal..$^{6}$

En su primer mensaje al concejo municipal, Graciela Contreras se impuso como objetivo mejorar el funcionamiento de los servicios ofrecidos por las distintas reparticiones municipales bajo un principio modernizador, aunque sin alejarse del ideario político del Frente Popular "que persigue en el orden edilicio el bienestar de los habitantes, a través de la organización de servicios públicos revestidos de prestigio y de eficiencia". ${ }^{37}$ Sobre el problema de las subsistencias su propuesta apuntaba a dejar de lado el rol pasivo que la legislación le asignaba a los municipios -según decía- por uno protagónico, con el objetivo declarado de asumir el vínculo entre productores, industriales y público consumidor, eliminando, de esta forma, los intermediarios..$^{8}$ En su mensaje, la alcaldesa llamó la atención sobre otro factor para explicar el alza de los productos agrícolas, como era la falta de planificación de las labores agrícolas y la existencia de terrenos baldíos en la periferia de la capital, "que hoy en su mayoría no se trabajan, con miras solo de especulación por sus posibilidades urbanísticas". ${ }^{29}$ Entre sus propuestas concretas estaba la creación de ferias libres, las que, sin embargo -señalaba-, debían desarrollarse en un marco de fomento crediticio a los pequeños productores para romper su dependencia de los consignatarios de la Vega Central.

A comienzos de marzo de I939, la alcaldesa resolvió crear, a modo experimental, las primeras ferias libres, determinándose que estarían bajo la dependencia del Departamento de Subsistencia en cuanto a reglamentos y fiscalización, contratando para ello un personal idóneo con los recursos de libre disposición..$^{40}$ En total fueron creadas cinco, en sectores periféricos del municipio de Santiago: avenida Matta, avenida ı de Julio, Calle Martínez de Rozas, avenida Santa Laura y la alameda de las Delicias (hoy avenida Bernardo O'Higgins). De acuerdo al decreto núm. 2019 del 9 de octubre de i939, cada una tenía asignado un día de la semana, con la excepción del día lunes que no funcionaban.

Es importante detenerse en la elección que se hizo de los lugares donde se instalarían las ferias. Tal como se observa en el mapa I, la Vega Municipal y el Mercado Central (sitios tradicionales de la oferta de productos agrícolas) se encontraban contiguas (núms. 6 y 7 en el mapa) y a orillas del río Mapocho, lo que hacía que las familias que habitaban en los sectores periféricos tuvieran dificultades en acceder diariamente a sus recintos. En este sentido, el hecho de que las ferias libres se distribuyeran principalmente en el sector sur y oeste de Santiago, los cuales albergaban zonas populosas, como el barrio de avenida Matta (núm. 5), la zona de avenida io de Julio (núm. 4) y la población Yungay (núm. 3), refleja el real interés que tenían las autoridades por facilitar el acceso a los productos agrícolas. De la misma manera, la feria ubicada en el sector norte (núm. 2) buscaba satisfacer la demanda de una zona más bien reducida de habitantes, pero con un perfil altamente popular y que se había asentado desde la época colonial al norte de río Mapocho. 
Mapa I. Sistema de abastos de la ciudad de Santiago. Década de r930

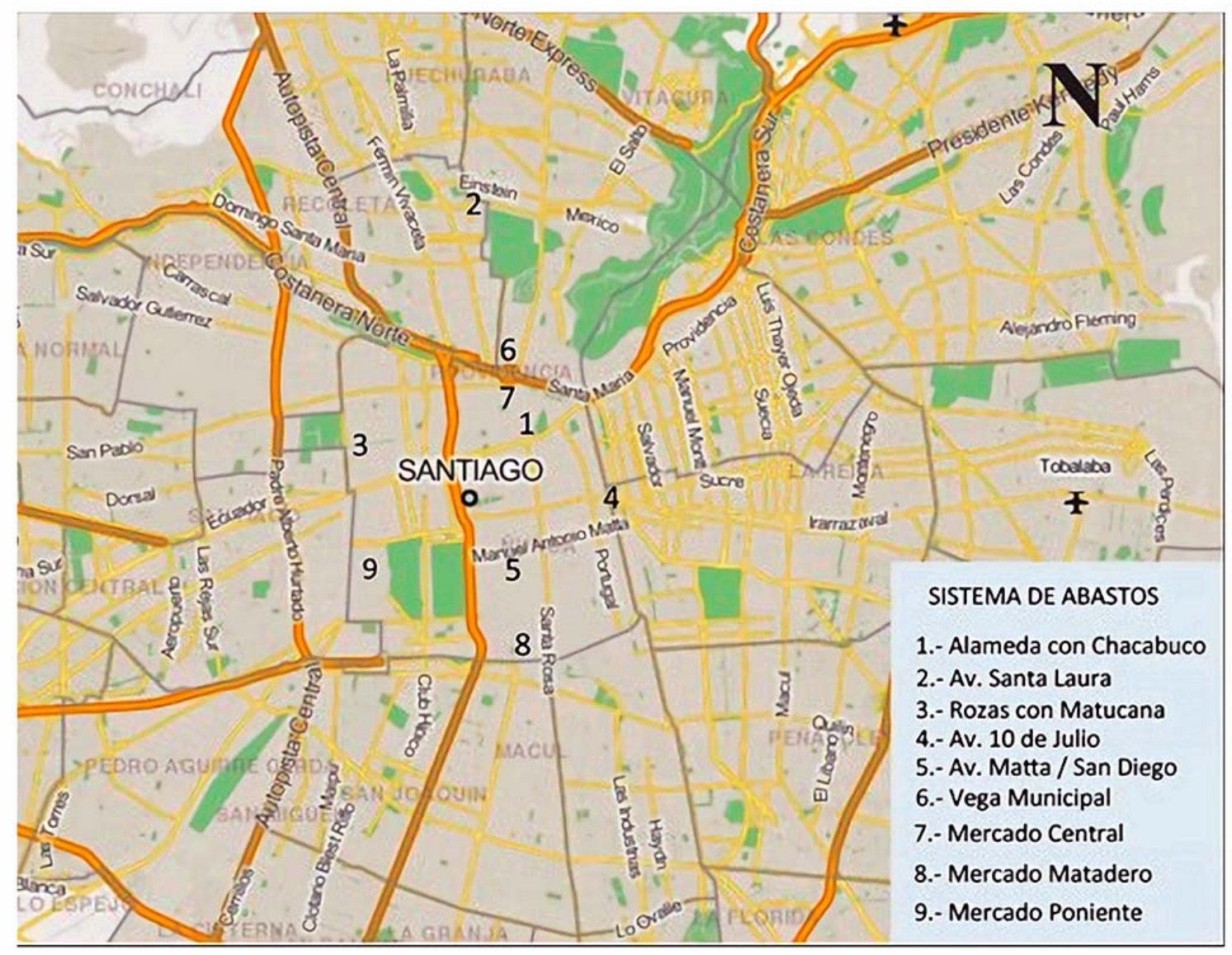

Fuente: Elaboración propia a partir de Boletín Municipal, 1936-1943. Las ferias libres se ubicaron en los primeros cinco puntos. Los puntos 6 al 9 corresponden al sistema tradicional de abastos de la capital. Se agradece al profesor Peter Bravo, Universidad de Valparaíso, por el apoyo técnico.

Si bien la creación de estas ferias tuvo el apoyo de algunos productores y gremios agrícolas, los comerciantes establecidos se opusieron tenazmente, con el argumento de que ellos no eran los responsables del alza de los alimentos, contando con el apoyo de los regidores Jorge Rivera y Santiago Poblete. ${ }^{4 I}$ Otros regidores demandaron la pronta discusión de un reglamento sobre las ferias libres, para subsanar el problema de autorizar el funcionamiento de estos establecimientos sin velar por las medidas de higiene y de fiscalización adecuadas. ${ }^{42}$ Es así como la alcaldesa aprobó con fecha 8 de septiembre de I939 un reglamento provisorio mientras se dictaba uno definitivo, el cual definía una feria libre como un espacio donde se realizaba el comercio directo entre los productores y consumidores, excluyendo, en consecuencia, a los intermediarios, aunque autorizaba la presencia de éstos en aquellos productos como carne y pescado que no podían ser vendidos por los propios productores. ${ }^{43}$ 
Desde un comienzo algunos regidores manifestaron su oposición a la creación de las ferias libres, argumentando tanto su baja efectividad en reducir el precio de los productos como los problemas de higiene que se observaban en ellas. ${ }^{44}$ En diversas ocasiones se reiteraron las denuncias sobre la presencia de comerciantes e intermediarios que se abastecían en la Vega o en el Mercado Central para luego ir a vender los productos a las ferias libres.

El regidor Jorge Rivera llamó la atención sobre lo inadecuado de reglamentar una actividad más bien puntual y reducida, la cual no debía institucionalizarse por ser considerada "contrario a la estética y a la dignidad de una ciudad como Santiago". ${ }^{55}$ Claramente, el regidor defendía el comercio establecido, principalmente los mercados municipales, los que según él habían hecho inversiones importantes, pagaban sus impuestos y recibían la competencia desleal de las ferias libres, proponiendo como alternativa que el concejo municipal estudiara la posibilidad de crear mercados municipales en sitios estratégicos de la capital.

En cuanto a la higiene, las posiciones se dividieron entre aquellas que rechazaban estos locales por afectar la salud de la población y las que, paradojalmente, apoyaban su funcionamiento, aunque fuera a costa de su salud. El siguiente diálogo de una sesión municipal de septiembre de I939 es fiel reflejo de las posiciones que marcaron el debate de esos años:

Regidor Juan Urzúa: "En la calle, en la Feria Libre, las moscas se pasean del guano de los caballos a la carne [...] Quizás, dando locales gratis a los carniceros y otras gangas puedan vender carne más barata en las Ferias Libres". Regidor Mena: "Es preferible que la gente coma aunque no sean tan higiénicos los locales en que se expenden los artículos de consumo a que no pueda comer. La cuestión es que compren barato". Regidor Rivera: "Aunque sea veneno...". Regidor Mena: "Sin comer se muere la gente de todas maneras...". "Debieran lisa y llanamente, no tener tantos escrúpulos...". 16

Más allá de estas polémicas, el año I939 marcó una consolidación de las ferias libres, por lo que el Departamento de Subsistencias en su memoria anual reconoció su aporte en facilitar el acceso más expedito a los alimentos, en especial, en aquellos lugares alejados de la Vega Central:

Sin constituir las Ferias Libres la solución del problema de las subsistencias que es complejo y está vinculado con todas las actividades económicas de la nación, hay que reconocer que ellas han mejorado apreciablemente el abastecimiento de la ciudad, facilitando un aprovisionamiento rápido y económico y creando fuertes núcleos de competencia comercial en sectores populares que antes carecían de ese beneficio por encontrarse muy distantes de los mercados municipales. ${ }^{47}$

Hacia I94I, el Departamento de Subsistencias podía señalar que: "Las condiciones higiénicas en general han mejorado, pues las verduras ya no se colocan sobre el piso sino en tableros sobre caballetes a una altura de $0.40 \mathrm{~m}$ de alto y últimamente se ha desalojado de la exposición, todos los vehículos del transporte animal". 48 
Prueba de la consolidación que tuvieron las ferias libres dentro del sistema general de abastos de la Municipalidad de Santiago es la regularidad de sus días de funcionamiento y el flujo de vehículos. Tal como lo muestra la tabla 2 hubo una regularidad en su funcionamiento, abriendo cada una un día a la semana y siendo las más importante por su flujo la ubicada en la calle Martínez de Rosas (sector San Pablo) y la de avenida Matta (sector Franklin), sectores populosos del norte y sur de Santiago, respectivamente.

Tabla 2. Estadísticas de Ferias Libres, 1940

\begin{tabular}{|c|c|c|}
\hline Feria & Días de funcionamiento & Ingreso de vehículos \\
\hline Avenida Matta & 52 & 7,880 \\
\hline Santa Lucía & $5^{2}$ & 2,912 \\
\hline Vivaceta & I2 & 379 \\
\hline Alameda & $5^{2}$ & 7,696 \\
\hline Avenida Io de Julio & 52 & 7,267 \\
\hline Martínez de Rosas & 53 & 3,289 \\
\hline Total & & $\mathbf{2 9 , 2 2 9}$ \\
\hline
\end{tabular}

Fuente: Boletín Municipal, núm. 4,808, 7 de noviembre de I94I.

Un paso importante en el desarrollo de las ferias libres se produjo con la aprobación de su reglamento definitivo en junio de I94I, el cual las definía como aquel comercio que se realizaba de forma directa entre productores y consumidores, con exclusión de los intermediarios, aunque el Departamento de Subsistencia de la Municipalidad podía autorizar la presencia de intermediarios en la venta de productos como carnes, pescados y mariscos. ${ }^{49}$ Para facilitar la venta de productos, se autorizaba el expendio directo en los automóviles que entraban a las ferias, aunque guardando las condiciones de higiene y salubridad. El reglamento determinó que los productores que querían vender en las ferias libres debían acreditar su calidad de tales con un certificado otorgado por una asociación gremial o sindical de productores. El artículo in del Reglamento estableció las obligaciones de los feriantes, entre las cuales estaban el clasificar los productos de acuerdo a su calidad, observar el lugar de llegada, mantener el orden y buen trato al público, así como la higiene de sus vehículos y productos. De esta forma, el reglamento no sólo vino a regularizar el funcionamiento de las ferias libres, sino que normó una actividad que lentamente transformaba el paisaje tradicional de la ciudad y a la cual ayudaron en su consolidación las propias luchas de los sectores populares, tal como veremos a continuación. 
Las luchas en torno a las ferias libres

Los efectos económicos de la Gran Depresión incentivaron la movilización y organización de diversos sectores de trabajadores, entre ellos los vinculados al comercio. Comerciantes, vendedores ambulantes y feriantes, entre otros, buscaron defender sus fuentes de trabajo, participar en el debate generado por el alza de las subsistencias y oponerse a alguna medida de las autoridades. Si bien existen pocos estudios que analicen las modalidades organizativas de los sectores de trabajadores en torno a las actividades comerciales del periodo, la prensa de la época registró muchos eventos donde éstos tomaron partido frente a las recién creadas ferias libres. Aunque no hubo muchas posiciones explícitas en contra de ellas, la mayoría de las críticas apuntaban al funcionamiento del sistema de abastos como causa principal en el alza del costo de la vida, proponiéndose desde distintos gremios mejoras en el funcionamiento de la Vega Central, evitando así el establecimiento de las ferias libres.

Un ejemplo de lo anterior es la Federación de Comerciantes en Subsistencias de Ferias y Mercados, creada en I934, y que reunía a locatarios del Matadero, de la Vega y del Mercado Central, siendo su órgano de expresión el periódico El Despertar. La Federación se constituyó como órgano de defensa frente a la carga tributaria impuesta por el municipio, aunque también como caja de ahorro para apoyar a los comerciantes en sus actividades. . $^{\circ}$ Además, contaba con un servicio de asistencia social que funcionaba como mutual. El aspecto sanitario fue asumido como bandera de lucha, tanto en su dimensión regenerativa de los espacios de venta, como en el cuidado de la salud de las personas que trabajaban en ellos, reclamando, por ejemplo, por los basurales cercanos al Matadero y los desperdicios y barro que inundaban la Vega Central. Además, lucharon en contra del alza en el precio de frutas y verduras, responsabilizando al carnet de crédito, el cual habilitaba a los comerciantes para operar en la Vega, y que servía para cobrar los costos del libro de contabilidad, el de estampillas, la libreta de sanidad y el impuesto a la renta, entre otros:

El escamoteo continúa periódicamente cuando llegan las bandadas de Inspectores Municipales a notificar de infracciones a los reglamentos. Traen la consiga de multar por cualquier motivo; lo importante es obtener nuevas entradas para la Ilustre Municipalidad y justificar esos empleos que no significan otra cosa que cuerpos legales para el abuso y el robo a desamparados compañeros nuestros, que la legislación actual los tiene en completo desamparo. ${ }^{\not 1}$

Por su parte, el Frente Único de Comerciantes Ambulantes y Estacionados comenzó desde marzo de 1936 a demandar en su órgano de difusión la inauguración de un Mercado Modelo, como solución a los problemas existentes en la Feria Municipal. ${ }^{22}$ En este caso, si bien no proponían la creación de una feria libre, reconocían los problemas del sistema tradicional de abastos de la capital. Otras organizaciones obreras sí demandaron la autorización para formar sus propias ferias libres, asegurando con ello una fuente de financiamiento, como lo hizo en I935 el Sindicato de Comerciantes en Ropa para vender las mercaderías de los asociados. 53 
En su momento, cuando la alcaldía de Valparaíso se propuso erradicar las ferias y el mercado ambulante del puerto, la Confederación de Trabajadores de Chile, máxima organización sindical del país, defendió su existencia, cuestionando las razones que habían dado las autoridades para erradicarlas, como eran las estéticas. Por el contrario, demandaba tener en cuenta la dimensión económica de esta actividad, donde trabajaban -según se señalaba- en 500 puestos establecidos 2,ooo jornaleros en verano y r,ooo en invierno:

La feria libre es en estas circunstancias una válvula reguladora que impide la especulación desmedida de los artículos de primera necesidad, y podemos constatar que los precios de las mercaderías que allí se expenden dan las posibilidades de pequeñas economías a los miles de hogares que en ella se surtan, lo que se justifica la existencia con las ioooo o más personas tanto de la clase obrera y clase media que constantemente acuden los miércoles y sábado de diferentes puntos de esta ciudad y localidades vecinas. ${ }^{44}$

En julio de 1939, los chacareros de Conchalí, Quilicura, Guanacos, Colina, Peñalolén, Nuñoa y otros sectores cercanos a la capital se organizaron en la Asociación de Chacareros de Santiago, demandando abrir las dependencias de la Vega para que ellos pudieran vender sus productos de forma directa, sin los consignatarios como intermediaros, junto con no limitar el ingreso de vehículos con productos de chacarería. Si bien esto no suponía una posición contraria a las ferias libres, proponía incluir reformas al funcionamiento de la Vega Municipal, manteniendo este espacio como el principal centro expendedor de frutas y verduras de la capital.

Sin embargo, hubo sectores que se mostraron contrarios al funcionamiento de las ferias libres, argumentando que los problemas en el alza del costo de la vida se debían más a problemas estructurales que al funcionamiento de la Vega, y que las ferias que se habían creado no habían ayudado a bajar los precios de frutas y verduras. El Sindicato de Comerciantes en Subsistencias de Ferias y Mercados Municipales se movilizó en junio de I939, recibiendo el apoyo de los regidores Jorge Rivera y Santiago Poblete, los cuales criticaron que la Municipalidad hubiese optado por crearlas afectando con ello el desarrollo del comercio establecido. ${ }^{56}$ Se resolvió formar una comisión de dirigentes de todo el comercio con el fin de estudiar la solución al problema de las ferias libres. Una de las primeras acciones de esta comisión fue entrevistarse con la alcaldesa de Santiago, donde se le hizo llegar un memorial de rechazo a su funcionamiento, señalando que no cumplían con el objetivo de bajar los precios porque los vendedores adquirían los productos en la misma Vega Municipal, violando el espíritu de la normativa: "Como única solución del problema, proponen los comerciantes la supresión inmediata de las Ferias Libres, que traería la tranquilidad a muchas personas que trabajan desde hace tiempo en las ferias [municipales] y a los miles que de ellas dependen". ${ }^{57}$

El 23 de octubre 1939, luego de aprobado el funcionamiento de las ferias por parte de la Municipalidad de Santiago, se formó el Comité Único de Sindicatos de Ferias y Mercados. Al parecer representaba a comerciantes minoristas de la Vega, Matadero y Mercado Central, que no tenían una posición político-partidista pero que se mostraban dispuestos a colaborar con la alcaldesa Graciela Contreras en sus intentos por abaratar el costo de los alimentos. Sin embargo, 
manifestaban un rechazo al funcionamiento de las ferias libres porque "no llenan las finalidades para las cuales fueron creadas, ni menos para aliviar la carestía de las subsistencias como ha quedado demostrado desde su creación". .8

A comienzos de I940, y luego de que las ferias libres parecían haberse consolidado como espacios de compra-venta, los comerciantes de la Vega, del Matadero, del Mercado y los chacareros de los alrededores de Santiago firmaron un memorial solicitando su reforma o eliminación. Entre las razones que se daban, estaba el hecho de que los chacareros no concurrían a ellas, lo que obligaba a los comerciantes a proveerse en la misma Vega Municipal, adquiriendo productos de segunda categoría y muy poco variados. ${ }^{59}$ Pese a la obligación de tener una placa o certificado de autentificación de la calidad de chacarero, los comerciantes calculaban que sólo el io \% de los vendedores de las ferias lo eran.

El precio de frutas y verduras fue uno de los principales argumentos que se tuvieron para apoyar o criticar el funcionamiento de las ferias libres. En el caso de la prensa nacional, local y obrera se expusieron variados argumentos en este sentido, aunque no existen informes rigurosos que nos permitan determinar si hubo una baja o no en los productos que se vendían en las ferias. Por ejemplo, el periódico de tendencia obrera Frente Popular inició a fines de i 939 una campaña en su contra, argumentando, precisamente, que no habían servido para bajar los precios. Por el contrario, se señalaba que los productos se habían encarecido, dando como ejemplo el precio de las papas, las que se vendían a \$0.80 y \$0.90 “cuando no debían estar a más de \$0.50".

Un artículo de 1939, bajo el seudónimo de Ariosto, criticaba la mala prensa que tenían las ferias libres, desnudando los diversos intereses existentes en torno a ellas:

Unos protestan porque con ellas se disminuyen algunas problemáticas entradas municipales y aplican un criterio utilitario; otros protestan porque ensucian algunas veredas y aplican un criterio de policía de aseo; otros, en fin, porque no hay control suficiente y aplican un criterio sanitario.

Consideraba que todos esos cuestionamientos podían ser solucionados ofreciendo espacios cerrados, bajo techo y con estricta fiscalización. Sin embargo, le parecía extraño que se acusara a las ferias libres de subir los precios, tal como los comerciantes de la Vega y Mercado Central denunciaban:

La verdad que el argumento, y sobre todo en boca de quien lo hace, no puede ser más peregrino. Si las ferias libres encarecen la vida, ¿qué mejor para ellos?; nadie comprará en las ferias libres y ellos obtendrán pingües utilidades. Hay argumentos que denotan, o un candor fabuloso en quienes los formulan, o la creencia en ellos de que la opinión pública está formada por "gente de las chacras".

Con ocasión de la celebración del primer aniversario de las ferias libres (febrero de i940), la prensa informó de una actividad en apoyo de la alcaldesa Graciela Contreras organizado por el denominado "Sindicato de Ferias Libres", ${ }_{33}$ organización que se hizo conocida por enfrentarse, 
durante i94I y I942, a los embates del comercio establecido. Si bien diversos informes de sanidad de I94I y I942 cuestionaron el funcionamiento de las ferias libres, en especial por el ingreso de caballos y la venta en las veredas, ${ }_{4}$ lo concreto es que las autoridades municipales no tuvieron la intención de eliminarlas tal como lo demandaba el comercio formal. Si a comienzos de r943, por ejemplo, se determinó prohibir en ellas la venta de carnes, pescados y mariscos, esta medida tendió a regular el ingreso de comerciantes a sus dependencias y garantizar mejores condiciones de higiene, por lo cual no significó una gran limitante para su desarrollo. ${ }^{65}$ De esta forma las ferias libres lograron con el tiempo consolidarse como espacios de expendio de frutas y verduras alternativos a la Vega Central.

\section{Conclusiones}

En el presente artículo se han querido trazar los principales problemas y críticas formuladas al sistema de abastos de la ciudad de Santiago a partir de la Gran Depresión. Si bien este proceso de crisis que afectó al conjunto de países latinoamericanos y del resto del mundo ha sido estudiado desde distintas perspectivas, en esta investigación hemos querido profundizar en los serios problemas de acceso a los alimentos que se dio en el periodo. Los cuestionamientos al sistema de abastos se orientaron a las modalidades de venta y el peso que tenían los consignatarios en la Vega Municipal y Mercado Central, lo que impedía una venta libre de los productos agrícolas.

En este sentido, en el cruce de los registros estadísticos que mostraban el alza en el costo de la vida, por una parte, y el desarrollo del comercio como un nuevo espacio de oportunidad de empleos (formales e informales), por otra, las disputas en torno a lo permitido y prohibido adquirieron un nuevo sentido en estos años.

En 1939, y luego de diversos ensayos que se dieron en distintas ciudades, la alcaldesa de Santiago, Graciela Contreras, aprobó la creación de las primeras cinco ferias libres, las que tenían por objetivo diversificar la oferta de productos agrícolas. Las posiciones en torno a las ferias sintetizaron un amplio clamor de críticas a las condiciones de funcionamiento del comercio tradicional, pero especialmente a un conjunto de prácticas y mecanismos de control ejercidos por vendedores y consignatarios que les garantizaba crecientes ganancias. Sin embargo, y pese a las críticas al funcionamiento del sistema de abastos, no todos los sectores fueron partidarios de las ferias libres, argumentando que las alzas se debían tanto a que éstas no estaban en condiciones de ofrecer productos más baratos, como a aspectos más estructurales, dentro de los cuales se mencionaban la especulación de terrenos y los problemas de producción y comercialización del agro.

Cuando el decreto de i94I reglamentó definitivamente el funcionamiento de las ferias libres, la disputa por su creación se trasladó a las condiciones exigidas para que no actuaran deslealmente con el comercio establecido. En concreto, este reglamento vino a formalizar un comercio cuya existencia no estaba del todo regulada, ofreciendo un reconocimiento jurídico a los feriantes, en la calidad de sujetos que tenían derechos y deberes. Pero, además, ayudó a empoderar las propias prácticas de los feriantes, que vieron en el reglamento una base de legitimación a sus 
organizaciones gremiales y la instancia que echó a andar una serie de protocolos de fiscalización, funcionarios y recursos que sería difícil de desinstalar en el futuro.

A partir de 1943, las páginas del Boletín Municipal mostraron mensualmente cómo las ferias libres se habían transformado -posiblemente desde fines de 1942- en otra fuente de entradas para el municipio, $\frac{6}{6}$ a través de los derechos que pagaban los chacareros que ingresaban sus carretelas y vehículos para vender sus productos en unas ferias libres que habían dejado, en parte, de ser libres.

\section{Referencias hemerográficas}

Boletín Municipal de la ciudad de Santiago, 1936-1943.

El Diario Ilustrado, Santiago, I935.

El Grito, Santiago, 1936.

El Mercurio, Santiago, 1936-I939.

Estadística Chilena, Santiago, I938.

Frente Popular, Santiago, I939-1940.

La Hora, Santiago, I935-1939.

La Opinión, Santiago, 1936-1937-1938.

Referencias bibliográficas

AGUILAR-RODRÍGUEZ, Sandra. "Cooking Modernity: Nutrition Policies, Class, and Gender in I940s and I950s Mexico City". The Americas 64(2) (2007): 177-205.

“Alimentando la nación: género y nutrición en México (I940-1960)". Revista de Estudios Sociales (29) (2008): 28-40.

ARMUS, Diego. Avatares de la medicalización en América Latina, 1870-1970. Buenos Aires: Lugar Editorial, 2005.

BARBOSA, Mario. El trabajo en la calle. Subsistencia y negociación política en la ciudad de México a comienzos del siglo XX. Tesis de Doctorado en Historia. México: El Colegio de México, 2005.

BARONA, Josep L. La medicalización del hambre. Economía política de la alimentación en Europa, 1918-1960. Barcelona: Icaria, 2014. 
CUETO, Marcos, ed. Missionaries of Sciences: The Rockefeller Foundation and Latin America. Bloomington: Indiana University Press, 1994.

El regreso de las epidemias: saludy sociedad en el Perú del siglo XX. Lima: Instituto de Estudios Peruanos, 2000.

DRAGONI, Carlos y Burnet ETIENNE. L'alimentation populaire au Chili. Première enquête générale de 1935. Santiago de Chile: Imprenta Universo, 1938.

DRINOT, Paulo. "Food, Race and Working-class Identity: Restaurantes Populares and Populism in I930s Peru". The Americas 62(2) (2005): 245-270.

GÓMEZ, Juan Carlos. "Crisis, hambre y socialismo: Chile, I93I-1932. Andes (7) (1988): IoI-I59.

HENRÍQUEZ, Rodrigo. En "Estado sólido". Políticas y politización en la construcción estatal. Chile, 1920-1950. Santiago: Ediciones Universidad Católica, 2014.

INE. Chile XI Censo de Población, 1940. Santiago: Celade, r940.

INNESS, Sherrie A., ed. Kitchen Culture in America: Popular Representations of Food, Gender, and Race. Philadelphia: University of Pennsylvania Press, 200I.

LÓPEZ, Isidora. Las ferias libres de Santiago. Memoria de Prueba de Historia y Geografía. Santiago de Chile: Instituto Pedagógico, Universidad de Chile, i955.

OCHOA, Enrique. Feeding Mexico: The Political Uses of Food since 1910. Wilmington: Scholarly Resources, 2000.

PERNET, Corinne. "L'OIT et la question de l'alimentation en Amérique latine (I930-I950)". En L'Organisation internationale du travail. Origine, développement, avenir, ed. Isabelle LespinetMoret y Vincent Viet. Rennes: Presses Universitaires de Rennes, 20II.

"Developing Nutritional Standars and Food Policy: Latin American Reformers between the ILO, the League of Nations Health Organization, and the Pan-American Sanitary Bureau". En Globalizing Social Rights. The International Labour Organization and Beyond,dir. Sandrine Kott y Joëlle Droux, 249-26r. Ginebra: OIT, 20I3.

POHL-VALERO, Stefan. "Alimentación, raza, productividad y desarrollo. Entre problemas sociales, nacionales y políticas nutricionales internacionales, Colombia, I890-I950". En Aproximaciones a lo local y lo global: América Latina en la historia de la ciencia contemporánea, dir. G. Mateos y E. Suárez-Díaz, II5-I54. México: Centro de Estudios Filosóficos, Políticos y Sociales Vicente Lombardo Toledano, 2016.

RAMÓN, Armando. Santiago de Chile (1541-1991). Historia de una sociedad urbana. Santiago: Editorial Sudamericana, 2000. 
REED, Edwyn. "Ferias francas". En Antecedentes y trabajos del Primer Congreso Nacional de Alimentación Popular. Santiago: Imprenta Aurora, 1932, 348-350.

SALAZAR, Gabriel. Ferias libres: espacio residual de soberanía ciudadana. Santiago de Chile: Ediciones Sur, 2003.

VARGAS, Joel. Alimentar el cuerpo social: ciencia, dieta y control en México durante el Porfiriato. Tesis de Maestría en Filosofía de la Ciencia. México: Universidad Nacional Autónoma de México, 20II.

VERGARA, Ángela. "Los trabajadores chilenos y las Gran Depresión, 1932-1938". En La Gran Depresión en América Latina, coord. Paulo Drinot y Alan Knight, 73-Io8. México: Fondo de Cultura Económica, 2015.

YÁÑEZ, Juan Carlos. "Alimentación abundante, sana y barata. Los restaurantes populares en Santiago (1936-1942)". Cuadernos de Historia(45) (2016): II7-I42.

. "El problema de la alimentación: un enfoque desde las encuestas de nutrición. Chile, 1928-1938”. América Latina en la Historia Económica 24(I) (2017): 66-97.

ZÁRATE, María Soledad. Dar a luz en Chile. Siglo XIX: de la "ciencia" de hembra a la ciencia obstétrica. Santiago: Dibam, 2007.

\section{Notas}

I Este artículo forma parte del Proyecto FONDECYT (CONICYT-Chile) de Investigación Postdoctoral $\mathrm{N}^{\mathrm{o}}$ 316047 titulado "Inequidad alimentaria y estándar de vida. Las políticas de alimentación popular en el Chile de Entre Guerra: I920-1950". Marcos Cueto, dir., Missionaries of Sciences: The Rockefeller Foundation and Latin America (Bloomington: Indiana University Press, I994); Marcos Cueto, El regreso de las epidemias: salud y sociedad en el Perú del siglo XX (Lima: Instituto de Estudios Peruanos, 200o); Diego Armus, Avatares de la medicalización en América Latina, 1870-1970 (Buenos Aires: Lugar Editorial, 2005); María Soledad Zárate, Dar a luz en Chile. Siglo XIX: de la "ciencia" de hembra a la ciencia obstétrica (Santiago de Chile: Dibam, 2007).

2 Enrique Ochoa, Feeding Mexico: The Political Uses of Food since 1910 (Wilmington: Scholarly Resources, 2000); Mario Barbosa, El trabajo en la calle. Subsistencia y negociación política en la ciudad de México a comienzos del siglo XX (Tesis de Doctorado en Historia, El Colegio de México, 2005); Sandra Aguilar-Rodríguez, "Alimentando la nación: género y nutrición en México (I940I960)", Revista de Estudios Sociales (29) (2008): 28-40; Joel Vargas, Alimentar el cuerpo social: ciencia, dieta y control en México durante el Porfiriato (Tesis de Maestría en Filosofía de la Ciencia, Universidad Nacional Autónoma de México, 20II).

3 Sherrie. A. Inness, dir., Kitchen Culture in America: Popular Representations of Food, Gender, and Race. (Filadelfia: University of Pennsylvania Press, 200I); Paulo Drinot, "Food, Race and 
Working-Class Identity: Restaurantes Populares and Populism in I9zos Peru", The Americas 62(2) (2005): 245-270; Sandra Aguilar-Rodríguez, "Cooking Modernity: Nutrition Policies, Class, and Gender in Ig40s and I950s Mexico City. The Americas 64(2) (2007): I77205; Juan Carlos Yáñez, "Alimentación abundante, sana y barata. Los restaurantes populares en $\underline{\text { Santiago (I936-I942)", Cuadernos de Historia (45) (2016): II7-I42. }}$

4 Corinne Pernet, "L'OIT et la question de l'alimentation en Amérique latine (I930-I950)", en L'Organisation internationale du travail. Origine, développement, avenir, dir. Isabelle LespinetMoret y Vincent Viet (Rennes: Presses Universitaires de Rennes, 2ori); Corinne Pernet, "Developing Nutritional Standars and Food Policy: Latin American Reformers between the $\underline{\text { ILO, the League of Nations Health Organization, and the Pan-American Sanitary Bureau", }}$ en Globalizing Social Rights. The International Labour Organization and Beyond, dir. Sandrine Kott y Joëlle Droux (Ginebra: OIT, 20I3); Josep L. Barona, La medicalización del hambre. Economía política de la alimentación en Europa, 1918-1960 (Barcelona: Icaria, 20I4); Stefan Pohl-Valero, "Alimentación, raza, productividad y desarrollo. Entre problemas sociales, nacionales y políticas nutricionales internacionales, Colombia, I890-1950", en Aproximaciones a lo local y lo global: América Latina en la historia de la ciencia contemporánea, dir. G. Mateos y E. Suárez-Díaz, II5-I54 (México: Centro de Estudios Filosóficos, Políticos y Sociales Vicente Lombardo Toledano, 2or6).

5 Armando de Ramón, Santiago de Chile (1541-1991). Historia de una sociedad urbana (Santiago de Chile: Editorial Sudamericana, 2000).

6 Isidora López, Las ferias libres de Santiago, Memoria de Prueba de Historia y Geografía (Santiago de Chile: Instituto Pedagógico, Universidad de Chile, 1955).

7 Gabriel Salazar, Ferias libres: espacio residual de soberanía ciudadana (Santiago de Chile: Ediciones Sur, 2003).

8 Juan Carlos Gómez, "Crisis, hambre y socialismo: Chile, I93I-I932", Andes (7) (Ig88): IOII59; Ángela Vergara, "Los trabajadores chilenos y la Gran Depresión, I932-1938", en La Gran Depresión en América Latina, coord. Paulo Drinot y Alan Knight, 73-Io8 (México: Fondo de Cultura Económica, 2015).

9 INE, Chile XI Censo de Población, 1940 (Santiago de Chile: Celade), 45I.

Io El censo de 1930 comprendió el comercio en un sentido amplio, incluyendo tanto el comercio de mercancías, bienes raíces, crédito, bolsa de comercio, hoteles y comercio ambulante. El censo de 1940 separó el rubro comercio (incluyendo el comercio de mercancías y vendedores ambulantes) del de servicios (bienes raíces, crédito, bolsa de comercio, hoteles y cafetería). Esto explica el crecimiento de 53 mil trabajadores en el sector de comercio.

I Carlos Dragoni y Etienne Burnet, L'alimentation populaire au Chili. Premiére enquête générale de 1935 (Santiago de Chile: Imprenta Universo, 1938). 
I2 Juan Carlos Yáñez, "El problema de la alimentación: un enfoque desde las encuestas de nutrición. Chile, 1928-1938", América Latina en la Historia Económica 24(I) (2017): 66-97.

I3 Véase, por ejemplo, el decreto núm. 460 del i8 de marzo de I939, que regulaba la labor de los consignatarios, Boletín Municipal, núm. 4,327,26 de marzo de ig40.

14 Rodrigo Henríquez, En "Estado sólido". Políticas y politización en la construcción estatal. Chile, 1920-1950 (Santiago de Chile: Ediciones Universidad Católica, 2014).

I5 Boletín Municipal, núm. 4,697, 25 de junio de I94I, I,I88.

I6 La Hora, Santiago de Chile, 2i de diciembre de ig39.

17 La Opinión, Santiago de Chile, I5 de mayo de 1936.

I8 En la época existían cuatro grandes firmas exportadoras: Compañía Frutera Sudamericana; Juan Sarraf; Jaccard y Pérez; y Enrique Stricker. Véase La Opinión zo de septiembre de ig36.

I9 Boletín Municipal, núm. 3,158, 28 de abril de 1936, I,33.

20 Boletín Municipal, núm. 3,299, ig de octubre de 1936, 3,115. Con respecto al papel de los consignatarios en el alza de las papas, se puede señalar, por ejemplo, que en noviembre de i939 del total de ventas $(\$ 545,152)$, sólo el 40 \% se hizo en remate público. Véase Boletín Municipal núm. 4.520, ig de noviembre de ig40.

2i Boletín Municipal, núm. 3,155, 24 de abril de i936, I,294.

22 El regidor Ángel Faivovich sintetizaba la posición de estos sectores en la siguiente frase: "En realidad, como síntesis de esta opinión frente al problema agrario podemos concluir que mientras el Estado no encare en forma integral un plan de política agraria nada definitivo podrá hacerse", Boletín Municipal, núm. 3,158, 28 de abril de 1936, I, 328.

23 El Mercurio, Santiago de Chile, 23 de julio de ig36.

24 Boletín Municipal, núm. 3,292, g de octubre de 1936, 2,085-2,089.

25 La Opinión, Santiago de Chile, i4 de octubre de ig36.

26 El Mercurio, Santiago de Chile, 5 de septiembre de 1936.

27 El Mercurio, Santiago de Chile, 5 de septiembre ig36.

28 Edwyn Reed, "Ferias Francas", en Antecedentes y trabajos del Primer Congreso Nacional de Alimentación Popular (Santiago de Chile: Imprenta Aurora, 1932), 348-350. 
29 La Hora, Santiago de Chile, iz de noviembre de i937.

30 La Opinión, Santiago de Chile, in de diciembre de igz7.

3і La Unión de Feriantes de Valparaíso formaba parte de la Federación Sindical de Valparaíso, la cual agrupaba a cerca de cuarenta sindicatos de la zona, como el sindicato de peluqueros, el de la compañía de gas, de trabajadores del aseo de Viña del Mar, de panificadores y de suplementeros, entre otros. Véase La Opinión, Santiago de Chile, 29 octubre de ig37.

32 La Opinión, Santiago de Chile, ig de noviembre de ig37.

33 La Opinión, Santiago de Chile, ig de noviembre de ig37.

34 La Caja del Seguro Obrero ofreció el concurso de los chacareros de los "fundos en colonización" que tenía la institución. Véase La Hora, Santiago de Chile, 28 de septiembre de 1935 .

35 Graciela Contreras, esposa del senador socialista Oscar Schnake, era dirigente del movimiento femenino socialista y de acuerdo con la ley de elecciones municipales, la alcaldía de Santiago debía ser designada directamente por el presidente de la República.

36 Boletín Municipal, núm. 3,962, 5 de enero i939.

37 Boletín Municipal, núm. 4,0I0, 2 de marzo I939, 424.

38 Boletín Municipal, núm. 4,0io, 2 de marzo i939, 425.

39 Boletín Municipal, núm. 4,0I0, 2 de marzo i939, 426.

4o Boletín Municipal, núm. 4,016, g de marzo I939.

4I Los comerciantes estaban organizados en el Sindicato Profesional de Comerciantes en Subsistencias de Ferias y Mercados. Véase El Mercurio, Santiago de Chile, 2 de junio de 1939.

42 Boletín Municipal, núm. 4,134, I de agosto de ig39.

43 Boletín Municipal, núm. 4,170, I3 de septiembre de i939.

44 Boletín Municipal, núm. 4,177, 25 de septiembre I939.

45 Boletín Municipal, núm. 4,I77, 25 de septiembre i939, 2,205.

46 Boletín Municipal núm. 4,I77, 25 de septiembre de I939, 2,207.

47 Boletín Municipal, núm. 4,520, Ig de noviembre de I940, 2,21I.

Relaciones Estudios de Historia y Sociedad, vol. XL, núm 157, 2019 
48 Boletín Municipal, núm. 4,705, 4 de julio de ig41, 2,I84.

49 Boletín Municipal, núm. 4,69I, I8 de junio I94I, I,I3I.

5o El Despertar, Santiago de Chile, 26 de mayo de 1934.

5i El Despertar, Santiago de Chile, i2 de junio de i934.

52 El Grito, Santiago de Chile, primera quincena mayo I936.

53 El Diario Ilustrado, Santiago de Chile, i2 de marzo de i935.

54 La Opinión, Santiago de Chile, 29 de junio de I938.

55 El Mercurio, Santiago de Chile, 3 de julio de ig39.

56 El Mercurio, Santiago de Chile, 2 de junio de ig39.

57 Firmaban la Sociedad Nacional de Comerciantes, el Sindicato Profesional de Comerciantes de Ferias y Mercados de Santiago y el Frente Único de Comerciantes Ambulantes y Minoristas de la Feria Municipal. Véase El Mercurio, Santiago de Chile, 22 de junio de razg.

58 El Mercurio, Santiago de Chile, 23 de octubre de ig39.

59 Frente Popular, Santiago de Chile, 24 de febrero de ig40.

6o Frente Popular, Santiago de Chile, is de noviembre de igz39.

6r La Hora, Santiago de Chile, 7 de noviembre de igz?.

62 La Hora, Santiago de Chile, 7 de noviembre de ig39.

63 Frente Popular, Santiago de Chile, ig de febrero de ig40.

64 Boletín Municipal, núm. 4,958, g de mayo de ig42.

65 Boletín Municipal, núm. 5,153, 5 de enero de ig43.

66 En el informe presupuestario del año ı942, se señala en el ítem Ingreso Fuera de Presupuesto "Derechos Ferias Libres", seguido de la suma de 128,286 pesos. En el mismo informe la recaudación domiciliaria de aseo era de 40,00o pesos. Véase Boletín Municipal, núm. 5, I88, is de febrero de $1943,3,604$. 


\section{Juan Carlos Yáñez Andrade}

Doctor en Historia, EHESS-Paris. Adscripción: Universidad de Valparaíso. Líneas de investigación: Historia del Estado Social; Historia de las instituciones internacionales; Historia de la alimentación y nutrición. Entre sus publicaciones más recientes se encuentran La OIT en América del Sur. El comunismo y los trabajadores chilenos (1922-1932). Santiago: U. Alberto Hurtado, 20r6; "Alimentación abundante, sana y barata. Los restaurantes populares en Santiago (1936I942)". Cuadernos de Historia (45) (2016): II7-I42; "El problema de la alimentación: un enfoque desde las encuestas de nutrición. Chile, I928-1938". América Latina en la Historia Económica (I) (2017): 66-97. 